OPEN ACCESS

Edited by:

Daniela Minerdi,

University of Turin, Italy

Reviewed by:

Marcela Claudia Pagano

Federal University of Minas Gerais,

Brazil

Bruno Tomio Goto,

Federal University of Rio Grande do

Norte, Brazil

*Correspondence:

Sidney Luiz Stürmer

sturmer@furb.br

Specialty section:

This article was submitted to

Fungi and Their Interactions,

a section of the journal

Frontiers in Microbiology

Received: 19 April 2020 Accepted: 23 November 2020

Published: 12 January 2021

Citation:

Stürmer SL and Kemmelmeier K

(2021) The Glomeromycota in the Neotropics.

Front. Microbiol. 11:553679. doi: 10.3389/fmicb.2020.553679

\section{The Glomeromycota in the Neotropics}

\author{
Sidney Luiz Stürmer* and Karl Kemmelmeier
}

Laboratory of Mycorrhiza, Department of Ciências Naturais, Universidade Regional de Blumenau (FURB), Blumenau, Brazil

Arbuscular mycorrhizal fungi (AMF-Glomeromycota) are a group of soil fungi with a widespread occurrence in terrestrial ecosystems where they play important roles that influence plant growth and ecosystem processes. The aim of this paper is to reveal AMF distribution in the Neotropics based on an extensive biogeography database with literature data from the last five decades. All four orders and 11 families were reported in the Neotropics. 221 species $(69 \%$ of the total number of species for the phylum) were registered in the Neotropics pertaining to 37 genera. Acaulospora, Glomus, Scutellospora, and Funneliformis were the most speciose genera and represented by 47, 29, 15, and 13 species, respectively. Seventy-six species were originally described from Neotropics, which represents $24 \%$ of the total diversity of Glomeromycota. The most representative families were Gigasporaceae, Ambisporaceae, and Acaulosporaceae with $89 \%, 80 \%$, and $79 \%$ of species within each family detected in the Neotropics, respectively. AMF were detected in 11 biomes and 52 ecological regions in 19 countries. Biomes with the largest number of species were Tropical and Subtropical Moist Forests (186 species), Tropical and Subtropical Dry Broadleaf forests (127 species), and Tropical and Subtropical Grasslands (124 species), and Jaccard's similarity among them was 53-57\%. Mean annual temperature and precipitation were not correlated with total AMF species richness. The Neotropics biomes shelter a large amount of the total diversity of Glomeromycota and studies of occurrence of these fungi should be encouraged considering their importance in maintaining terrestrial ecosystems.

Keywords: biogeography, biomes, South America, arbuscular mycorrhizal fungi, species distribution, Central America

\section{INTRODUCTION}

Fungi in the phylum Glomeromycota include the arbuscular mycorrhizal fungi (AMF) which are associated with vascular plants and thalloid bryophytes (Brundrett and Tedersoo, 2018) and Geosiphon pyriformis associated with the cyanobacteria Nostoc (Schussler et al., 1994). Fossil evidences place the origin of AMF for at least 400 million years in the Devonian period where they 
were associated with the first vascular land plants from the Rhynie chert formation (Remy et al., 1994; Dotzler et al., 2006, 2009). Considering the lack of host specificity and the long geological time they have to disperse around the globe, it is not surprising that AMF are widely widespread in terrestrial ecosystems forming the arbuscular mycorrhizal association with $72 \%$ of plants species (Brundrett and Tedersoo, 2018) in all biogeographic realms and biomes (Stürmer et al., 2018). Distribution of AMF evidenced from molecular data (Davison et al., 2015) and spore morphology (Stürmer et al., 2018) reveals low levels of endemism for AMF species. There are currently 317 species of AMF morphologically described (International Culture Collection of Glomeromycota [CICG], 2020) and distributed in four orders (Diversisporales, Glomerales, Archaeosporales, and Paraglomerales), 11-16 families, and 39-49 genera depending on the classification system followed (Wijayawardene et al., 2020).

The Neotropical biogeographic realm, as delimited by the Sclater-Wallace system, includes Central America (up to central Mexico), the Caribbean Islands, and South America, and it has fascinated naturalists and biologists for its high biodiversity (Chazot et al., 2019). A revision of the Wallace biogeographical realms by Holt et al. (2013) includes the Caribbean islands and part of Central America in the Panamanian realms. The Neotropics are distributed across the North American, the Caribbean, and the South America tectonic plates (Antonelli and Sanmartín, 2011). As part of the Gondwana, the South American continent started to separate from Africa ca. 125 million years ago, developed its own biota during $>90$ million years while drifting west and relatively isolated from other continents, and became connected with North America by the Isthmus of Panama at 3.5 million years ago (Gentry, 1982; Brown and Lomolino, 2008). Diversification in the Neotropics was also affected by event of uplifting, including the formation of the Andean cordilleras which modified its climate and landscape (Hoorn et al., 2010), and by climatic fluctuations in the Pleistocene (Prance, 1973). Indeed, Neotropics includes 11 out of 14 terrestrial biomes recognized by Olson et al. (2001): Desert and Xeric Shrublands (DXS), Mangroves (MAN), Mediterranean Forests (MED), Montane Grasslands and Shrublands (MON), Temperate Broadleaf and Mixed Forests (TMF), Temperate Grasslands (TGS), Tropical and Subtropical Dry Forests (SDF), Tropical and Subtropical Grasslands (SGS), Tropical and Subtropical Moist Forests (SBF), Flooded Grasslands (FGS), and Tropical and Subtropical Coniferous Forests (SCF).

Occurrence and diversity of Glomeromycota in Neotropical biomes have been investigated, mainly in Brazil, Argentina, and Chile (Cofré et al., 2019). These studies emphasized comparison among distinct habitats (Castillo et al., 2005; Stürmer and Bellei, 1994; Carvalho et al., 2012; Pereira et al., 2014), variation along environmental gradients (Lugo et al., 2008; Silva et al., 2014; Coutinho et al., 2015; Velázquez et al., 2016a,b), and seasonal variation of AMF population (Lugo and Cabello, 2002; Escudero and Mendoza, 2005; Stürmer and Siqueira, 2011). Moreover, diversity of AMF was assessed in agricultural ecosystems (Siqueira et al., 1989; Carrenho et al., 2001; Aguilera et al., 2014), mining sites (Schneider et al., 2013; Souza et al., 2013), and habitats under revegetation (Cuenca and Lovera, 1992). Checklists of Glomeromycota species have been produced for some ecoregions within the Neotropics like the Brazilian Cerrado (Jobim et al., 2016), Caatinga (Goto et al., 2010), and Atlantic rainforest (Jobim et al., 2018). Cofré et al. (2019) compiled results of AMF species occurrence in South America based on 110 articles and recorded 186 species belonging to 9 families and 24 genera. Authors emphasized the occurrence of AMF species within the distinct ecoregions in South America and recorded Amazonia, Atlantic Forest, Caatinga, and Chaco as the regions with the highest number of species detected. For Brazil, Maia et al. (2020) recorded a total of 192 AMF species in 38 genera and 15 families, which represents $60 \%$ of the total richness described for Glomeromycota. Most of the studies and species recorded in Brazilian territory were from the Atlantic rainforest, the Cerrado savanna, and the Caatinga dry forest, emphasizing that Brazilian ecosystems are important repository for this group of soil fungi.

In this work, a systematic biogeography method (Parenti and Ebach, 2009) was used to analyze data from a Glomeromycota biogeography database (BD) that includes information of AMF distribution obtained from published literature, accession database from living culture collections, and species description (Stürmer et al., 2018). Our goal is to provide an updated checklist of AMF species and genera detected in the Neotropics and elucidate AMF distribution patterns according to the major Neotropical biomes.

\section{MATERIALS AND METHODS}

The $\mathrm{BD}$ is organized with four main classes of information: taxonomic (data on order, family, genus and species), geographic (data on biogeographical realm, biomes, ecoregions, continent, country, state, location, plant host, latitude and longitude, hemisphere, and climatic zone), environmental (data on soil $\mathrm{pH}, \mathrm{P}, \mathrm{N}, \mathrm{C}$, and organic matter, and temperature, precipitation and altitude), and origination category (indicating whether data came from species description, records from INVAM (International Culture Collection of (Vesicular) Arbuscular Mycorrhizal Fungi, Morgantown, WV, United States) and CICG (International Culture Collection of Glomeromycota, Blumenau, SC, Brazil), or other published manuscript) (Stürmer et al., 2018). Biogeographic realms are considered based on Holt et al. (2013) and biomes and ecoregions recognized according to Olson et al. (2001).

For this paper, we searched the BD for the term "Neotropics" and "Panamanian" as they cover the Neotropical realm according to Wallace, the concept being followed in this special issue. After that, we grouped the records by biomes and generated a list of families, genera, and AMF species occurring in each biome. A measure of frequency of occurrence of a species was calculated based on the number of records in the database and proportion of families among biomes investigated based 
on the number of species pertaining to a given family. Venn diagram was used to depict number of AMF species unique and shared among selected biomes which were most extensively surveyed for AMF. Jaccard's index was used to determine the similarity of AMF species among biomes and calculated using PAST (Hammer et al., 2001).

Longitude and latitude data from manuscripts that surveyed AMF communities were used to obtain data of mean annual temperature and precipitation from WorldClim ${ }^{1}$. We then investigated the relationship of both climate data with total species richness and number of species belonging to Glomeraceae, Gigasporaceae, and Acaulosporaceae using linear models.

We found some taxonomic conundrum during our study that must be clarified. First, we followed Bentivenga and Morton (1995) who recognized only five species of Gigaspora. Second, species with gigasporoid mode of spore formation were considered to be part of the family Gigasporaceae as recommended by Redecker et al. (2013). Third, Fuscutata aurea was considered in our species list since it was not considered by Redecker et al. (2013) when species of Fuscutata were transferred to Dentiscutata. Fourth, we followed the arrangement proposed by Bills and Morton (2015) for Ambispora. Fifth, we followed the proposal of Walker et al. (2017) to conserve the name Rhizophagus but some species are referred herein as Rhizoglomus only because of the lack of nomenclatural combination to the genus Rhizophagus, since molecular phylogenetic data clearly put these species in the Rhizophagus clade.

\section{RESULTS}

A total of 5178 records (out of 10,961) were recovered of the biogeography database for the Neotropical realm, which originated from 178 articles published and 62 accessions of INVAM and CICG (Supplementary File 1). The number of AMF species recorded for the Neotropics is 221, which represents 69\% of the 317 species (International Culture Collection of Glomeromycota [CICG], 2020) described up to date for the phylum Glomeromycota (Table 1). All orders (Archaeosporales, Paraglomerales, Diversisporales, and Glomerales) were detected and taxa pertained to 37 genera in the families Glomeraceae (76 species), Acaulosporaceae (47 species), Gigasporaceae (51 species), Diversisporaceae (15 species), Paraglomeraceae (7 species), Archaeosporaceae (5 species), Pacisporaceae (6 species), Claroideoglomeraceae (6 species), Ambisporaceae (4 species), Pervetustaceae (1 species), and Sacullosporaceae (1 species). Entrophospora infrequens (Incertae sedis) was also recorded but not assigned to any family, following the recommendation of Redecker et al. (2013). The most frequent species occurring in the Neotropics based on the number of records were Claroideoglomus etunicatum (185 records), Acaulospora scrobiculata (212 records), Acaulospora mellea (164 records), and Ambispora leptoticha (146 records)

${ }^{1}$ https://www.worldclim.org/data/bioclim.html
(Figure 1). Acaulospora mellea, Claroideoglomus etunicatum, and Ambispora leptoticha were the only species detected in ten biomes and 63 species were detected in only one biome. Seventy-six species of AMF (24\% of the phylum) were described from type specimens found in the Neotropics (Supplementary Table 1). We also found six heterotypic synonym based on specimens collected from the Neotropics (Supplementary Table 1).

Studies were conducted in most countries of South America but there are no records in the BD from Uruguay, Paraguay, Guiana, Suriname, French Guiana, and El Salvador. The Caribbean islands are represented in the BD by Cuba, Jamaica, Guadeloupe, and Martinique. The highest numbers of records in the $\mathrm{BD}$ were from Brazil, Argentina, Mexico, and Colombia, from where $182,77,87$, and 77 species were recorded, respectively (Supplementary Table 2).

AMF species were registered from all 11 biomes occurring in the Neotropical realm, and distribution of families based on the number of species differed among biomes (Figure 2). Six families were detected for DXS and MON, and seven families for SCF, while nine to ten families were reported for other biomes. Pervetustaceae and Sacullosporaceae were recorded only for SDF and SBF, respectively, while Archaeosporaceae, Diversisporaceae, and Pacisporaceae were not detected for FGS and MON. Paraglomeraceae and Archaeosporaceae were not recorded for SCF and DXS, respectively. Species in FGS pertained mainly to Acaulosporaceae and Gigasporaceae which together accounted for $68.4 \%$ of the total number of species. Glomeraceae accounted for $32.6 \%$ to $55.6 \%$ of species in DXS, MAN, MED, MON, SBF, and SCF, while for TMF, TGS, SDF, and SGS families Glomeraceae, Acaulosporaceae, and Gigasporaceae were more evenly distributed.

Biomes TMF, TGS, SDF, SGS, and SBF harbored the largest number of species and 26 species were shared among them (Figure 3). These species pertained to 13 genera distributed in the families Ambisporaceae, Archaeosporaceae, Acaulosporaceae, Gigasporaceae, Diversisporaceae, Claroideoglomeraceae, and Glomeraceae. Species shared by two, three, and four biomes ranged mostly from 0-9; however, this number ranged from 18 to 28 when SGS and SBF were included in the comparison (Figure 3). The highest number of exclusive species was detected in SBF (43 species) followed by SDF (14 species) and SGS (6 species). Species were detected in 52 different ecoregions within biomes in the Neotropics (Supplementary Table 3). Ecoregions Caatinga, Cerrado, and Serra do Mar coastal forest harbored 98, 92, and 86 species, respectively, and were the ecoregions with the highest number of species recorded (Supplementary Table 3).

We investigated patterns of similarity of AMF species composition among Neotropical biomes using Jaccard's index (Table 2). Similarity among most biomes ranged from $9 \%$ to $43 \%$ (Table 2), and the average similarity among all biomes was $25 \%$. The largest number of AMF species was recorded for SBF (186 species), SDF (127 species), and SGS (124 species) (Table 1), and similarity among these biomes ranged from 53 to $57 \%$. Similarity of MED and MON with other biomes 
TABLE 1 | Families and species of Glomeromycota occurring in Neotropics biomes.

\section{Families/species}

DXS MAN

MED

MON

\section{Paraglomeraceae}

Paraglomus albidum (C. Walker \& L.H.

Rhodes) Oehl, F.A. Souza, G.A. Silva \&

Sieverd.

Paraglomus bolivianum (Sieverd. \&

Oehl) Oehl \& G.A. Silva

Paraglomus brasilianum (Spain \& J.

Miranda) J.B. Morton \& D. Redecker

Paraglomus laccatum (Błaszk.) C.

Renker, Błaszk. \& Buscot

Paraglomus lacteum (S.L. Rose \&

Trappe) Oehl, F.A. Souza, G.A. Silva \&

Sieverd.

Paraglomus occidentale

Corazon-Guivin, G.A. Silva \& Oehl

Paraglomus occultum (C. Walker) J.B.

Morton \& D. Redecker

Paraglomus pernambucanum Oehl,

C.M. Mello, Magna \& G.A. Silva

Pervetustaceae

Pervetustus simplex Błaszk., Chwat,

Kozłowska, Crossay, Symanczik \&

Al-Yahya'ei

\section{Ambisporaceae}

Ambispora gerdemannii (S.L. Rose,

B.A. Daniels \& Trappe) R.J. Bills \& J.B. Morton comb. nov.

Ambispora leptoticha (N.C. Schenck \& G.S. Sm.) R.J. Bills \& J.B. Morton

Ambispora nicolsonii (C. Walker, L.E.

Reed \& F.E. Sanders) Oehl, G.A. Silva,

B.T. Goto \& Sieverd.

Ambispora reticulata Oehl \& Sieverd.

Archaeosporaceae

Archaeospora ecuadoriana A. Schüßler \& C. Walker

Archaeospora myriocarpa (Spain,

Sieverd. \& N.C. Schenck) Oehl, G.A.

Silva, B.T. Goto \& Sieverd.

Archaeospora schenkii (Sieverd. \& S.

Toro) C. Walker \& Schüßler

Archaeospora trappei (R.N. Ames \&

Linderman) J.B. Morton \& D. Redecker

Archaeospora undulata (Sieverd.)

Sieverd., G.A. Silva, B.T. Goto \& Oehl

\section{Acaulosporaceae}

Acaulospora alpina Oehl, Sýkorová \&

Sieverd.

Acaulospora aspera Corazon-Guivin,

Oehl \& G.A. Silva

Acaulospora baetica Palenz., Oehl,

Azcón-Aguilar \& G.A. Silva

Acaulospora bireticulata F.M. Rothwell

\& Trappe

Acaulospora brasiliensis (B.T. Goto,

L.C. Maia \& Oehl) C. Walker, M. Krüger

\& A. Schüßler

Acaulospora capsicula Blaszk.

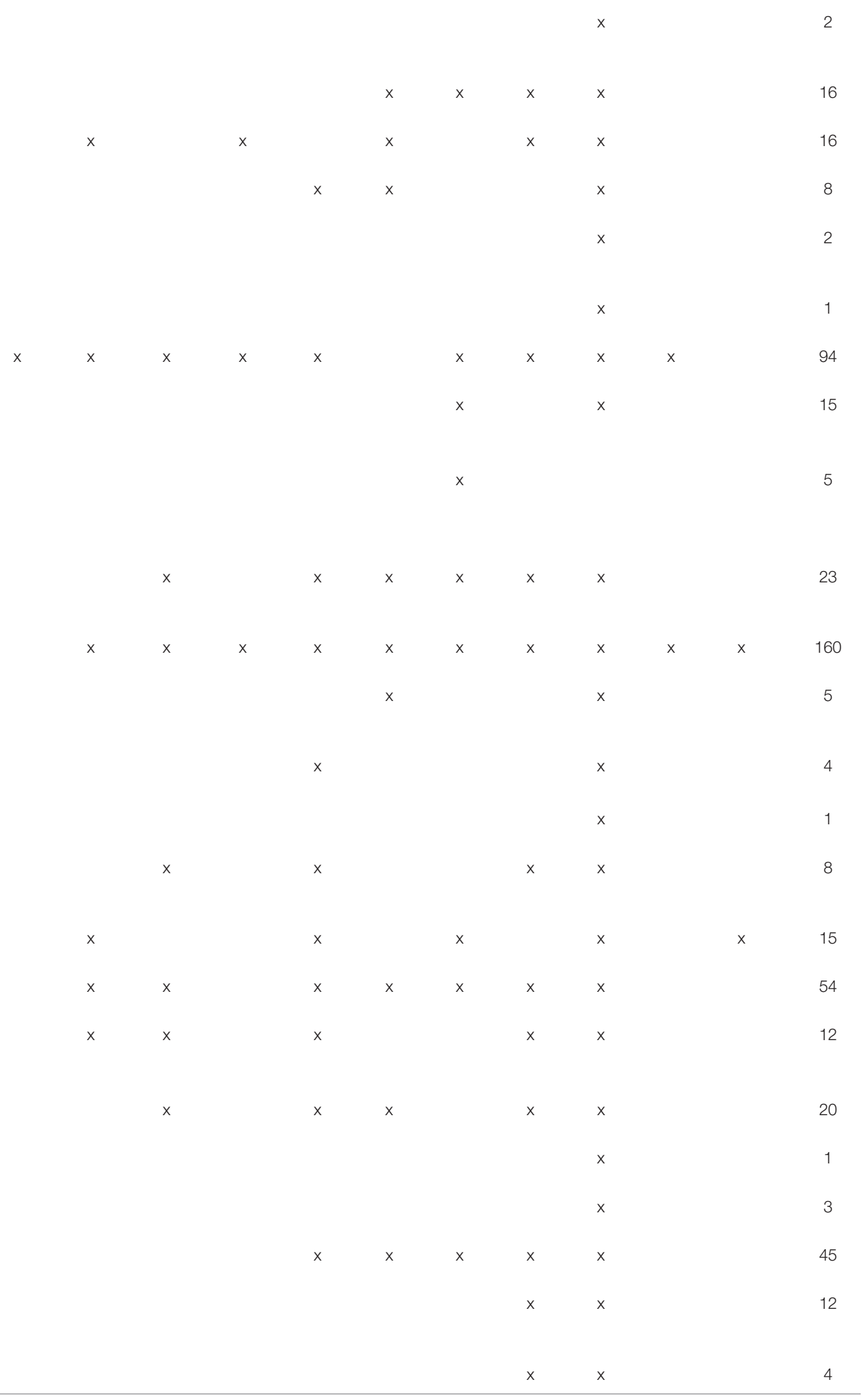

(Continued) 
TABLE 1 | Continued

\section{Families/species}

Acaulospora cavernata Błaszk.

Acaulospora colombiana (Spain \& N.C. Schenck) Kaonongbua, J.B. Morton \&

Bever

Acaulospora colossi P.A. Schultz, Bever \& J.B. Morton

Acaulospora delicta C. Walker, C.M.

Fief. \& Bliss

Acaulospora denticulata Sieverd. \& S.

Toro

Acaulospora dilatata J.B. Morton

Acaulospora elegans Trappe \& Gerd.

Acaulospora endographis B.T. Goto

Acaulospora entreriana M.S. Velázquez \& Cabello

Acaulospora excavata Ingleby \& C.

Walker

Acaulospora foveata Trappe \& Janos

Acaulospora gedanensis Błaszk.

Acaulospora herrerae Furrazola,

B.T.Goto, G.A.Silva, Sieverd. \& Oehl

Acaulospora ignota Blaszk., Góralska,

Chwat \& Goto

Acaulospora kentinensis (C.G. Wu \&

Y.S. Liu) Kaonongbua, J.B. Morton \&

Bever

Acaulospora koskei Błaszk.

Acaulospora lacunosa J.B. Morton

Acaulospora laevis Gerd. \& Trappe

Acaulospora longula Spain \& N.C.

Schenck

Acaulospora mellea Spain \& N.C.

Schenck

Acaulospora minuta Oehl, Tchabi,

Hount., Palenz., I.C. Sánchez \& G.A.

Silva

Acaulospora morrowiae Spain \& N.C.

Schenck

Acaulospora nivalis Oehl, Palenz., I.C.

Sánchez, G.A. Silva \& Sieverd.

Acaulospora papillosa C.M.R. Pereira \&

Oehl

Acaulospora paulineae Błaszk.

Acaulospora polonica Błaszk.

Acaulospora punctata Oehl, Palenz.,

I.C. Sánchez, G.A. Silva, C. Castillo \&

Sieverd.

Acaulospora reducta Oehl, B.T. Goto \&

C.M.R. Pereira

Acaulospora rehmii Sieverd. \& S. Toro

Acaulospora rugosa J.B. Morton

Acaulospora scrobiculata Trappe

Acaulospora sieverdingii Oehl,

Sýkorová, Błaszk. \& G.A. Silva

Acaulospora spinosa C. Walker \&

Trappe

\section{DXS}

MAN

MED

MON

TMF

MF TGS

TGS

SDF

$x$

$x$

$x$

$x+x$

SGS

SBF

$\begin{array}{cccc}\text { BF } & \text { FGS } & \text { SCF } & \text { Record } \\ \times & & & 16 \\ \times & x & & 69\end{array}$

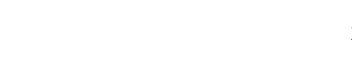

$x$

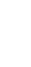

$x$

$x$

$x$
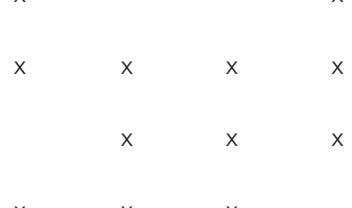

x

x

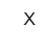

18

11

3

14

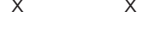

\section{x}$$
x
$$
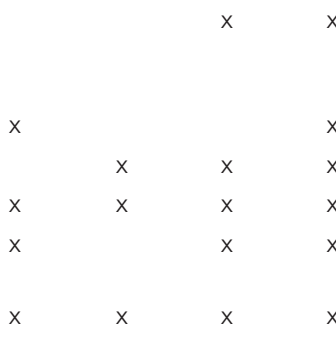

X

23

$x \quad x$

7

106

59

178

5

$x$

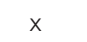

$x$

$x$

135

$x$

2

4

24

$x$
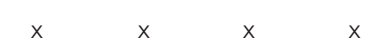

$x$

2

$x$

10

$x+x$

\begin{tabular}{|c|c|c|c|c|c|c|c|c|c|}
\hline & & & $x$ & $x$ & $x$ & $x$ & & & 13 \\
\hline & & & $x$ & $x$ & $x$ & $x$ & & & 75 \\
\hline & $x$ & & & & $x$ & $x$ & & & 10 \\
\hline & $x$ & $x$ & $x$ & $x$ & $x$ & $x$ & $x$ & $x$ & 234 \\
\hline$x$ & & $x$ & & $x$ & $x$ & $x$ & & & 13 \\
\hline & $x$ & $x$ & $x$ & $x$ & $x$ & $x$ & $x$ & $x$ & 129 \\
\hline
\end{tabular}

(Continued) 
TABLE 1 | Continued

\section{Families/species}

DXS

MAN

MED

MON

Acaulospora spinosissima Oehl,

Palenz., I.C. Sánchez, Tchabi, Hount. \&

G.A. Silva

Acaulospora spinulifera Oehl, V.M.

Santos, J.S. Pontes \& G.A. Silva

Acaulospora splendida Sieverd.,

Chaverri \& I. Rojas

Acaulospora sporocarpia S.M. Berch

Acaulospora thomii Błaszk.

Acaulospora tuberculata Janos \&

Trappe

Acaulospora verna Błaszk.

Acaulospora walkeri Kramad. \& Hedger

Incertae saedis

Entrophospora infrequens (I.R. Hall)

R.N. Ames \& R.W. Schneid.

\section{Diversisporaceae}

Corymbiglomus corymbiforme (Błaszk.)

Błaszk. \& Chwat

Corymbiglomus globiferum (Koske \& C.

Walker) Błaszk. \& Chwat

Corymbiglomus pacificum Oehl,

Medina, P. Cornejo, Sánchez-Castro,

G.A. Silva \& Palenz.

Diversispora aurantia (Błaszk., Blanke,

Renker \& Buscot) C. Walker \& A.

Schüßler

Diversispora eburnea (L.J. Kenn., J.C.

Stutz \& J.B. Morton) C. Walker \& A.

Schüßler

Diversispora pustulata (Koske, Fries, C.

Walker \& Dalpé) Oehl, G.A. Silva \&

Sieverd.

Diversispora spurca (C.M. Pfeiff., C.

Walker \& Bloss) C. Walker \& A.

Schüßler

Diversispora trimurales (Koske \&

Halvorson) C. Walker \& A. Schüßler

Diversispora versiformis (P. Karst.) Oehl,

G.A. Silva \& Sieverd.

Otospora bareae J. Palenzuela, N.

Ferrol \& Oehl

Redeckera fulva (Berk. \& Broome) C.

Walker \& A. Schüßler

Redeckera megalocarpum (D.

Redecker) C. Walker \& A. Schüßler

Redeckera pulvinatum (Henn.) C.

Walker \& A. Schüßler

Sieverdingia tortuosa (N.C. Schenck \&

G.S. Sm.) Błaszk., Niezgoda \& B.T.

Goto

Tricispora nevadensis (Palenzuela,

Ferrol, Azcón-Aguilar \& Oehl) Oehl,

Palenzuela, G.A. Silva \& Sieverd.

\section{Gigasporaceae}

Bulbospora minima Oehl, Marinho, B.T.

Goto \& G.A. Silva

$x$

$x$

$x$

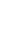

$x$
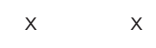

$x$

$x$

72

$\mathrm{x}$
$\mathrm{x}$

$x$

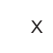

X

X

$x$

2

8

81 
TABLE 1 | Continued

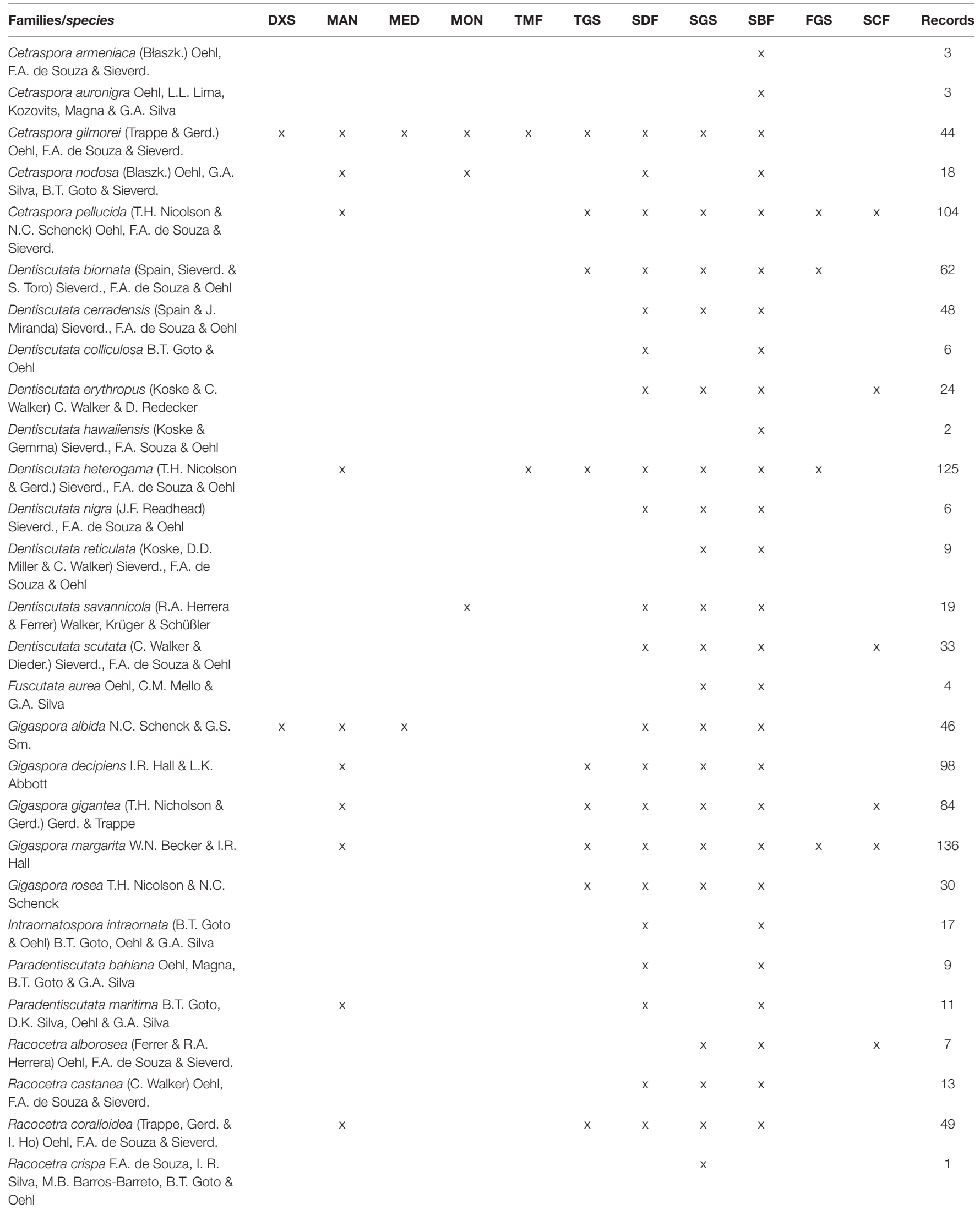


TABLE 1 | Continued

\section{Families/species}

Racocetra fulgida (Koske \& C. Walker) Oehl, F.A. de Souza \& Sieverd.

Racocetra gregaria (N.C. Schenck \&

T.H. Nicolson) Oehl, F.A. de Souza \&

Sieverd.

Racocetra minuta (Ferrer \& R.A.

Herrera) Oehl, F.A. de Souza \& Sieverd.

Racocetra persica (Koske \& C. Walker)

Oehl, F.A. de Souza \& Sieverd.

Racocetra tropicana Oehl, B.T. Goto \&

G.A. Silva

Racocetra verrucosa (Koske \& C.

Walker) Oehl, F.A. de Souza \& Sieverd.

Scutellospora alterata Oehl, J.S.

Pontes, Palenz., Sánchez-Castro \&

G.A. Silva

Scutellospora arenicola Koske \&

Halvorson

Scutellospora aurigloba (I.R. Hall)

C.Walker \& F.E. Sanders

Scutellospora calospora (T.H. Nicolson \& Gerd.) C. Walker \& F.E. Sanders

Scutellospora crenulata R.A.

Herrera-Peraza, Cuenca \& C. Walker

Scutellospora dipapillosa (C. Walker \&

Koske) C. Walker \& F.E. Sanders

Scutellospora dipurpurescens J.B.

Morton \& Koske

Scutellospora pernambucana Oehl, D.K

Silva, N. Freitas, L.C. Maia

Scutellospora projecturata Kramad. \&

C. Walker

Scutellospora rubra Stürmer \& J.B.

Morton

Scutellospora spinosissima C. Walker \&

Cuenca

Scutellospora striata Cuenca \& Herrera

Scutellospora tepuiensis Furrazola \&

Cuenca

Scutellospora tricalypta (R.A. Herrera \&

Ferrer) C. Walker \& F.E. Sanders

Scutellospora weresubiae Koske \& C.

Walker

\section{Pacisporaceae}

Pacispora chimonobambusae (C.G. Wu \& Y.S. Liu) Sieverd. \& Oehl ex C Walker, Vestberg \& Schüßler

Pacispora dominikii (Blaszk.) Sieverd. \& Oehl

Pacispora franciscana Oehl \& Sieverd.

Pacispora patagonica (Novas \&

Fracchia) C. Walker, Vestberg \&

Schüßler

Pacispora robigina Oehl \& Sieverd.

Pacispora scintillans (S. L. Rose \&

Trappe) Sieverd. \& Oehl ex C. Walker,

Vestberg \& Schüßler

\section{DXS}

MAN

MED

MON

$x$

TGS

$x$

SD

$x$

SGS

SBF

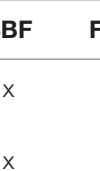

FGS

$\begin{array}{cc}\text { SCF } & \text { Records } \\ \times & 53 \\ & 31\end{array}$

$x$

$x$

$x \quad x \quad x$

$\begin{array}{lll} & x & x \\ x & x & x \\ x & x & x \\ x & x & \\ x & & \end{array}$

7

16

20

21

2

3

$x$

$x$

$x$

$x$

$x$

$x$

$\mathrm{X}$

17

$x$

$\mathrm{x}$

$x$

$x$

90

$x$

$x$

$x$

$x$

23

$x$

x

X

$x$

36

$x$

x

x

4

$x$
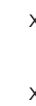

X

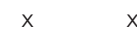

$x \quad x$

31

22

2

1

21

$x$

X

$x$

$x$

$x$

$x$

9

4

6 
TABLE 1 | Continued

\section{Families/species}

\section{Sacculosporaceae}

Sacculospora baltica (Błaszk., Madej \& Tadych) Oehl, Palenz., Sánchez-Castro, B.T. Goto, G.A. Silva \& Sieverd.

\section{Claroideoglomeraceae}

Claroideoglomus claroideum (N.C Schenck \& G.S. Sm.) C. Walker \& A. Schüßler

Claroideoglomus drummondii (Blaszk. \& C. Renker) C. Walker \& A. Schüßler Claroideoglomus etunicatum (W.N. Becker \& Gerd.) C. Walker \& A. Schüßler

Claroideoglomus lamellosum (Dalpé, Koske \& Tews) C. Walker \& A. Schüßler Claroideoglomus luteum (L.J. Kenn., J.C. Stutz \& J.B. Morton) C. Walker \& A. Schüßler

Claroideoglomus walker (Blaszk. \& C. Renker) C. Walker \& A. Schüßler

\section{Glomeraceae}

Dominikia aurea (Oehl \& Sieverd.) Blaszk. Chwat, G.A. Silva \& Oehl Dominikia bernensis Oehl, Palenz., Sánchez-castro \& G.A. Silva

Dominikia indica (Błaszk., Wubet \& Harikumar) Błaszk., G.A. Silva \& Oehl Dominikia iranica (Błaszk., Kovács \& Balázs) Błaszk., Chwat \& Kovács Dominikia minuta (Błaszk., Tadych \& Madej) Błaszk., Chwat \& Kovác Funneliformis badium (Oehl, Redecker \& Sieverd.) C. Walker \& A. Schüßler Funneliformis caesaris (Sieverd. \& Oehl) Oehl, G.A. Silva \& Sieverd.

Funneliformis caledonium (Nicolson \& Gerdemann) C. Walker \& A. Schüßler Funneliformis coronatum (Giovann.) C. Walker \& A. Schüßler

Funneliformis dimorphicus (Boyetchko \& J.P. Tewari) Oehl, G.A. Silva \& Sieverd.

Funneliformis fragilistratum (Skou \& I. Jakobsen) C. Walker \& A. Schüßler Funneliformis geosporum (T.H. Nicolson \& Gerd.) C. Walker \& A. Schüßler Funneliformis halonatum (S.L. Rose \& Trappe) Oehl, G.A. Silva \& Sieverd.

Funneliformis kerguelensis (Dalpé \& Strullu) Oehl, G.A. Silva \& Sieverd. Funneliformis monosporus (Gerd. \& Trappe) Oehl, G.A. Silva \& Sieverd.

Funneliformis mosseae (T.H. Nicolson \& Gerd.) C. Walker \& A. Schüßler

Funneliformis multiforum (Tadych \& Blaszk.) Oehl, G.A. Silva \& Sieverd. Funneliformis verruculosum (Blaszk.) C. Walker \& A. Schüßler

\section{DXS MAN}

MED

MON

TMF

TGS

SDF

SGS

SGS

SBF

FGS

SCF

Records

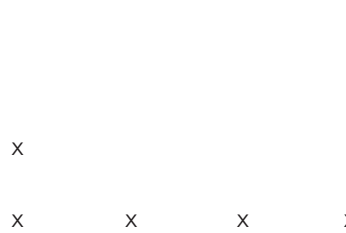


TABLE 1 | Continued

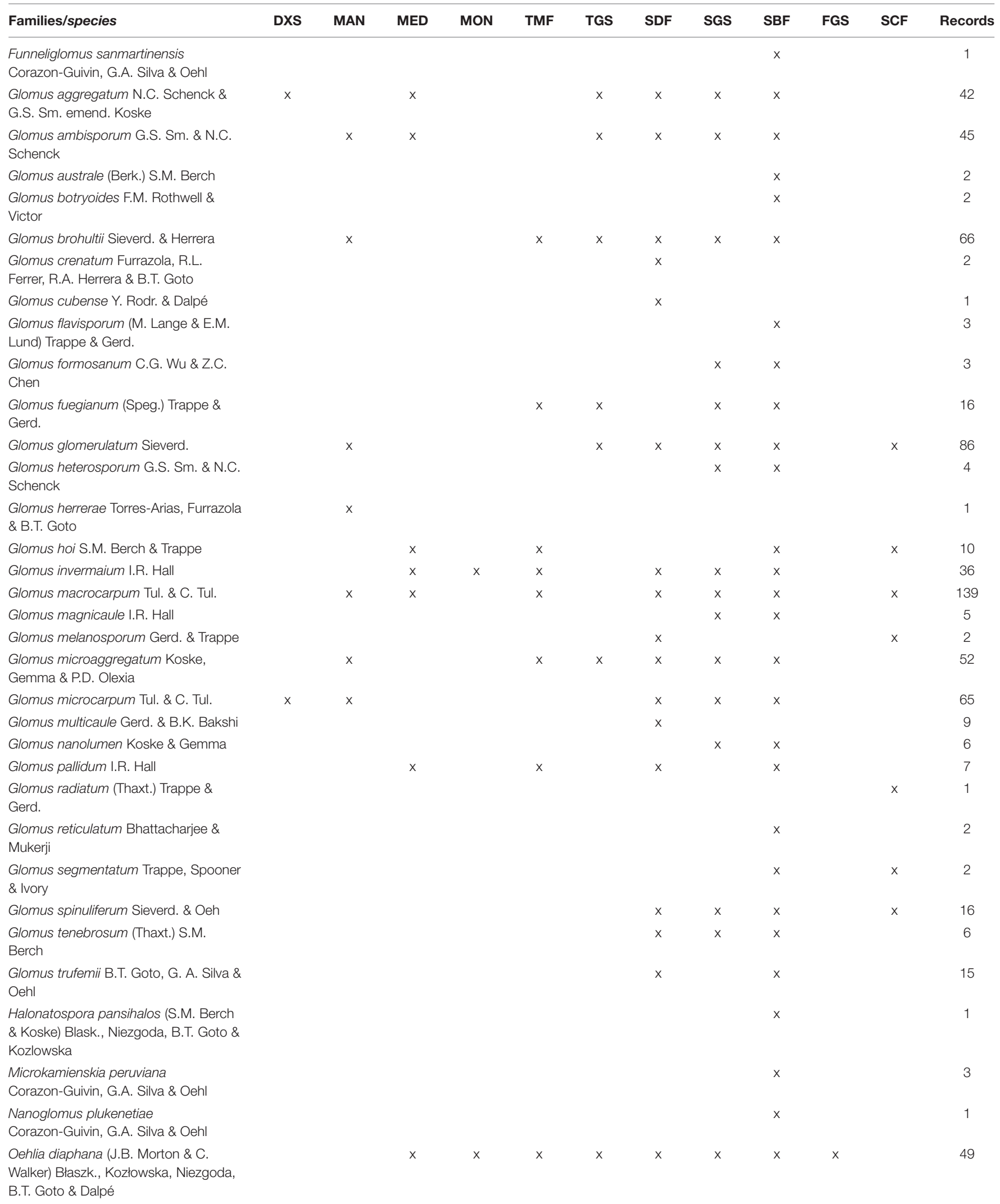


TABLE 1 | Continued

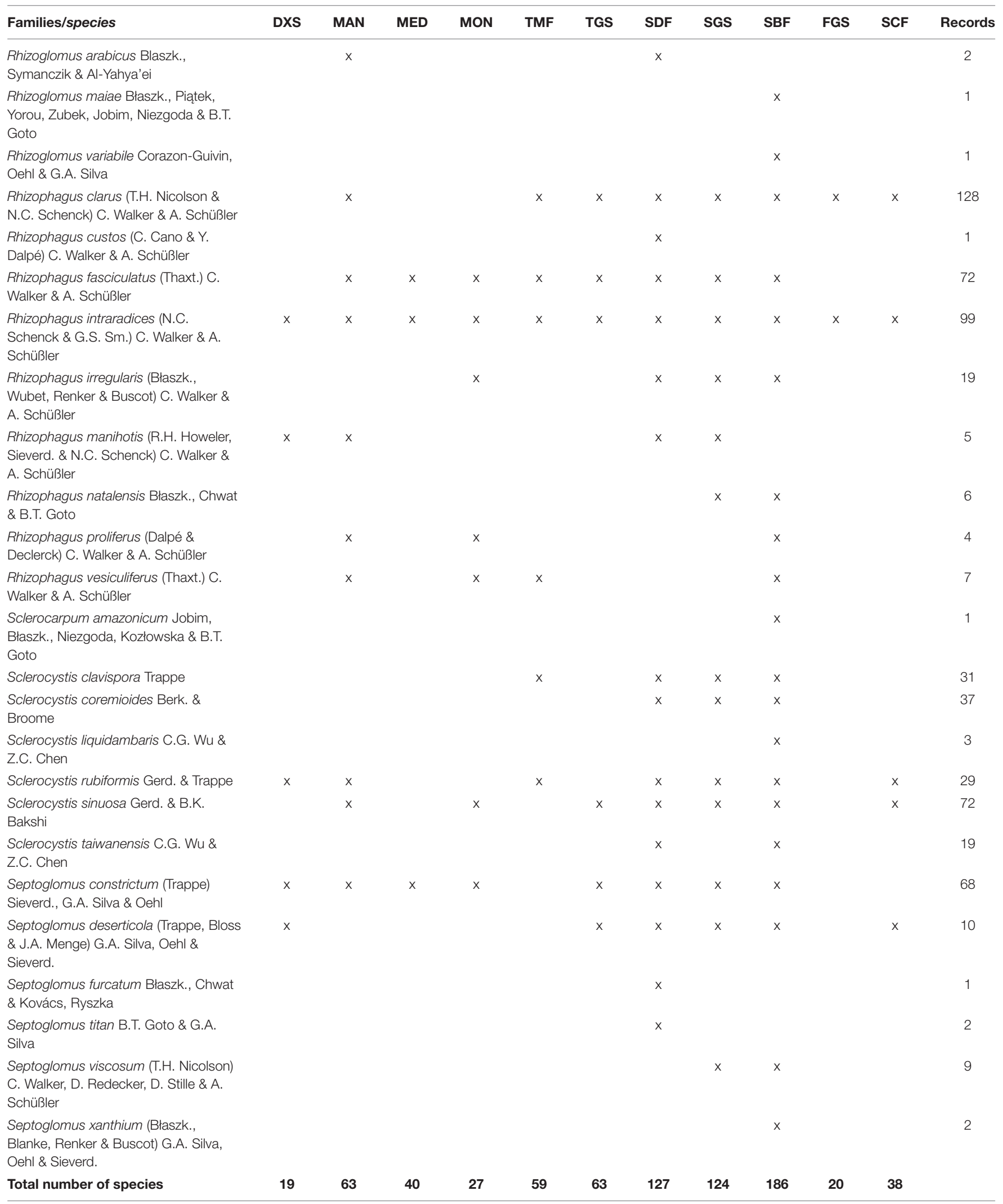

DXS, Deserts \& Xeric Shrublands; MAN, Mangroves; MED, Mediterranean Forests; Woodlands \& Scrub; MON, Montane Grasslands \& Shrublands; TMF, Temperate Broadleaf \& Mixed Forests; TGS, Temperate Grasslands, Savannas \& Shrublands; SDF, Tropical \& Subtropical Dry Broadleaf Forests; SGS, Tropical and Subtropical Grassslands; Savannas \& Shrublands; SBF, Tropical \& Subtropical Moist Broadleaf Forests; FGS, Flooded Grasslands \& Savannas; SCF, Tropical and Subtropical Coniferous Forests. 

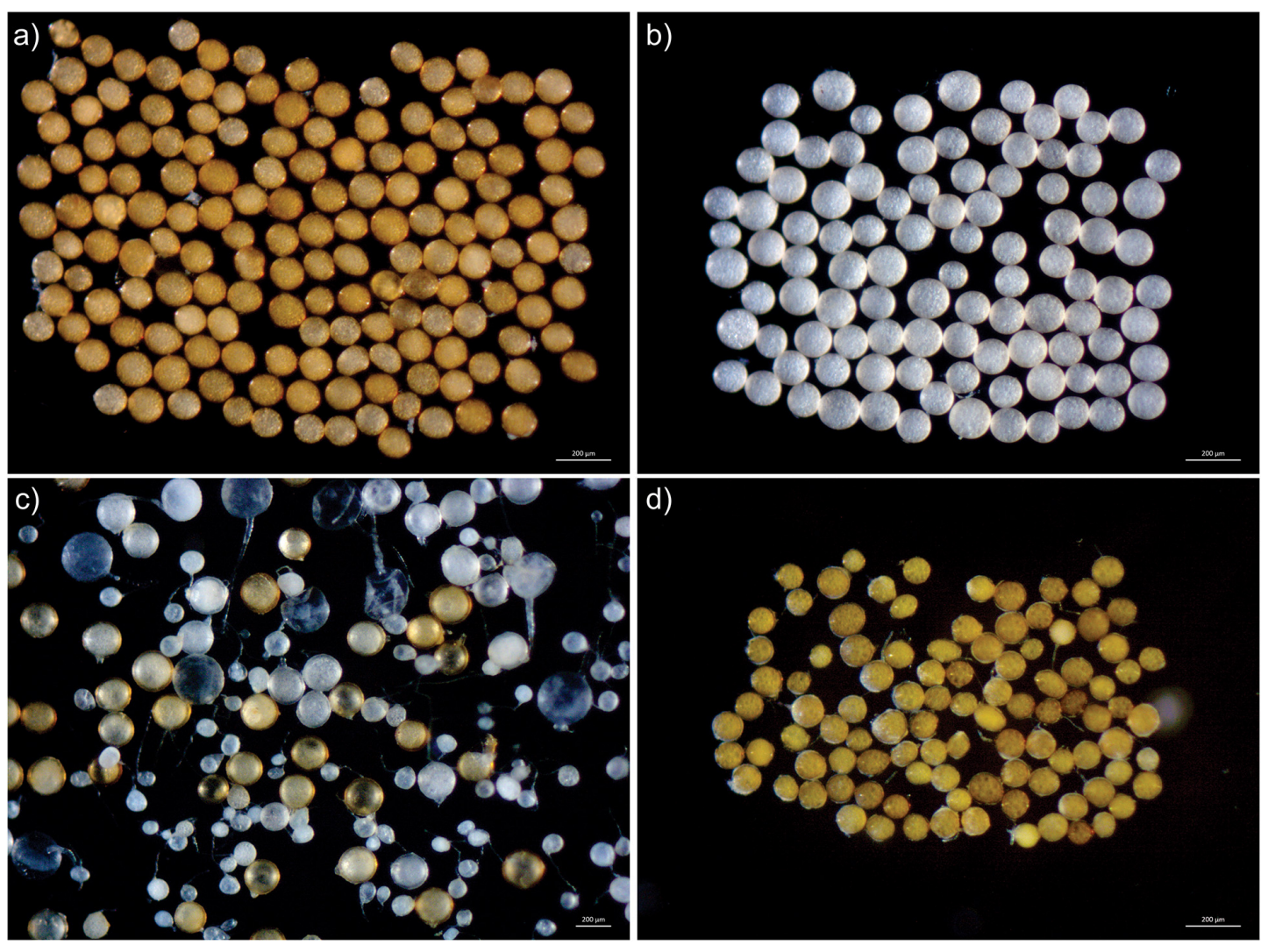

FIGURE 1 | Spores of the most frequent species of Glomeromycota in the Neotropics: (a) Acaulospora mellea, (b) Acaulospora scrobiculata, (c) Ambispora leptoticha, (d) Claroideoglomus etunicatum. Source: International Culture Collection of Glomeromycota (www.furb.br/cicg).

ranged from 13 to $26 \%$, except MED and TMF that shared $50 \%$ of species.

We found a very weak negative relationship between total number of AMF species and mean annual temperature and total precipitation; however, this relation was significant only for total precipitation (Figure 4). The linear models indicated that number of species in Gigasporaceae had a significant positive relationship with mean annual temperature and a significant negative relationship with total precipitation while number of species in Acaulosporaceae showed no significant relationship with both climatic data (Figure 4).

\section{DISCUSSION}

This study improves our knowledge on AMF distribution in the Neotropics following the contribution of Cofré et al. (2019) for South America and Maia et al. (2020) for Brazil. While Cofré et al. (2019) focused on reporting AMF species richness in distinct ecoregions in South America and Maia et al. (2020) focused on Brazilian floristic domains, our study converged to reveal distribution patterns among biomes occurring in the Neotropics. We found that 221 species of AMF have been registered from Neotropics, representing $69 \%$ of the total diversity of the phylum Glomeromycota. This result indicates that the Neotropical realm shelters a significant portion of the total diversity of the phylum, despite that some biomes and ecoregions are poorly surveyed for AMF species. For instance, most of the surveys carried in tropical forests in the Neotropics are from ecoregions pertaining to the Atlantic forest in the eastern region of Brazil while few studies were done in ecoregions of the Amazon basin. Desert and Xeric Shrublands, Flooded Grasslands, Mangroves, Mediterranean Forests, Montane Grasslands and Shrublands, and Tropical and Subtropical Coniferous Forests have very few studies on AMF occurrence compared to other biomes. Survey types of studies for AMF with a strong taxonomic basis should be encouraged in the Neotropics considering that some biodiversity hotspots are found in this realm (Myers et al., 2000) and new taxa have been discovered in the Neotropics in the last decade (e.g., Goto et al., 2012; Blaszkowski et al., 2013; Jobim et al., 2019). Moreover, investigation of the AMF community associated with native and economically important plants occurring in the Neotropics like Ilex paraguariensis and Araucaria angustifolia (Moreira et al., 2007) might reveal new and unreported AMF species.

All families and genera in Glomeromycota were represented in the Neotropics, even families like Sacculosporaceae and Pervetustaceae which are populated by two and one species, respectively, and described from Europe and Asia were recorded for this realm. Glomeraceae, Acaulosporaceae, and 


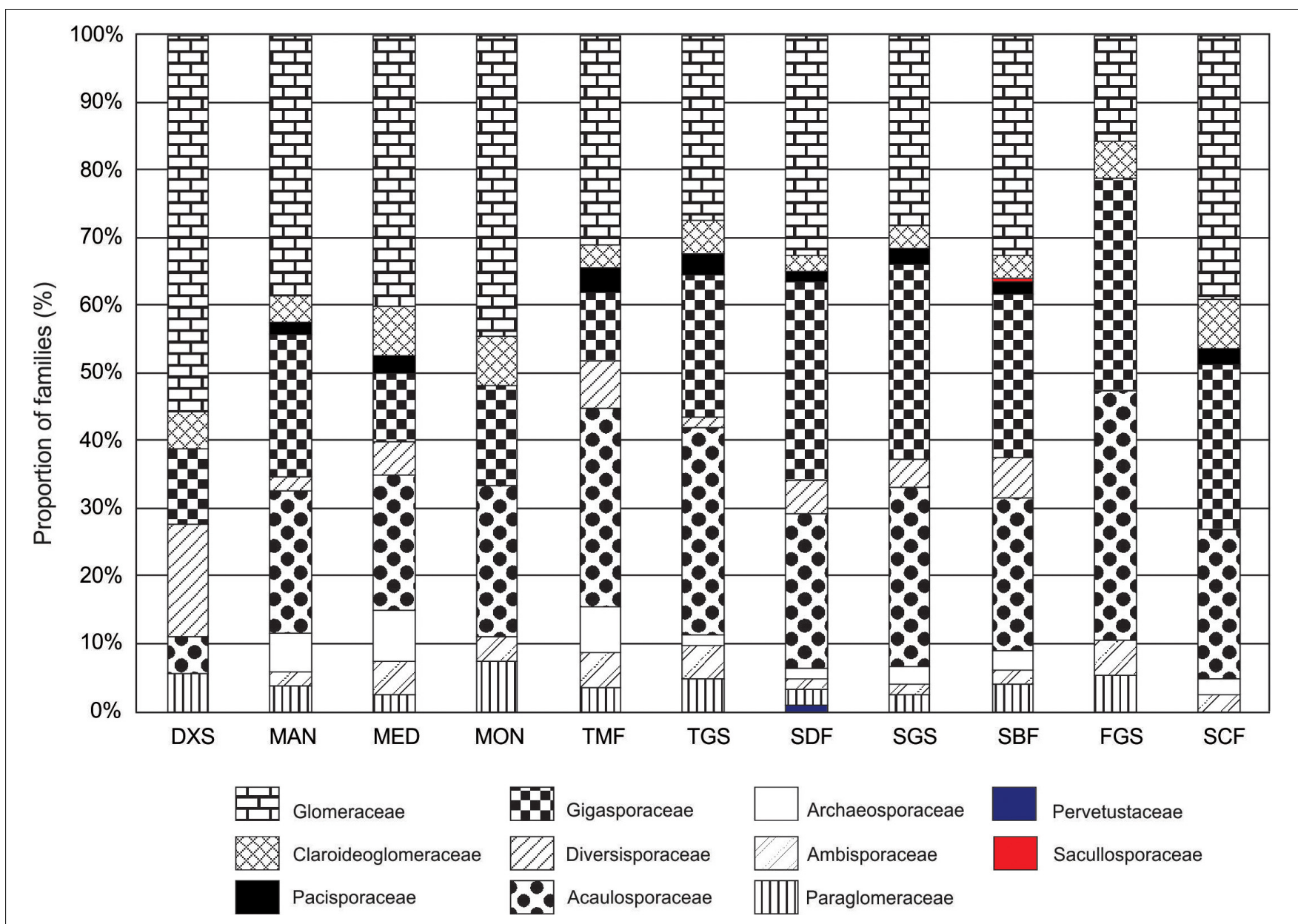

FIGURE 2 | Proportion of families (\%) in Glomeromycota based on the number of species per family in Neotropical biomes. DXS, Desert and Xeric Shrublands; MAN, Mangroves; MED, Mediterranean Forests, Woodlands \& Scrub; MON, Montane Grasslands \& Shrublands; TMF, Temperate Broadleaf \& Mixed Forests; TGS, Temperate Grasslands; Savannas \& Shrublands; SDF, Tropical \& Subtropical Dry Broadleaf Forests; SGS, Tropical and Subtropical Grasslands; Savannas \& Shrublands; SBF, Tropical \& Subtropical Moist Broadleaf Forests. FGS, Flooded Grasslands \& Savannas; SCF, Tropical and Subtropical Coniferous Forests.

Gigasporaceae could be considered the most representative families in the Neotropics considering the absolute number of species registered within each family. However, these families are the most speciose families within the phylum with 57123 species described followed by Diversisporaceae (34 species), while other families are formed by 1-9 species. Considering the widespread distribution of AMF species in global ecosystems (Davison et al., 2015; Stürmer et al., 2018), it is not surprising that these three families are the most representative in the Neotropics. We suggest that representativeness of supraspecific taxa should be investigated based on the proportion of species relative to the total number of species described for that taxa. For instance, when this approach is used, Gigasporaceae, Ambisporaceae, and Acaulosporaceae are the most representative families as $89 \%, 80 \%$, and $79 \%$ of the species within each family have been reported in the Neotropics. A drawback of this approach is for monospecific families and genera as their representativeness will be always $100 \%$ once the species has been registered.
Among the four most frequent species in the Neotropics detected herein, only Ambispora leptoticha was not included in the list of frequent species in South America compiled by Cofré et al. (2019). The possible reason for this is that these authors considered Ambispora appendicula, A. callosa, $A$. fecundispora, and $A$. jimgerdemannii as distinct species while we considered them to be synonymous with A. leptoticha (Bills and Morton, 2015). The four most frequent species detected in the Neotropics are not exclusive of this biogeographic realm as they were recorded in other five continents and considered to be cosmopolitan (Stürmer et al., 2018). Conversely, it is interesting that $29 \%$ of the species detected herein were found in only one biome within the Neotropics, which might be an indicative that they are rare species or associated with a particular soil or climate condition. Interesting is that the most frequent species belongs to three genera (Ambispora, Acaulospora, and Claroideoglomus), from three distinct families and orders. This suggests that AMF communities are composed by co-occurring fungi with different life-history strategies (Hart and Reader, 2002). 


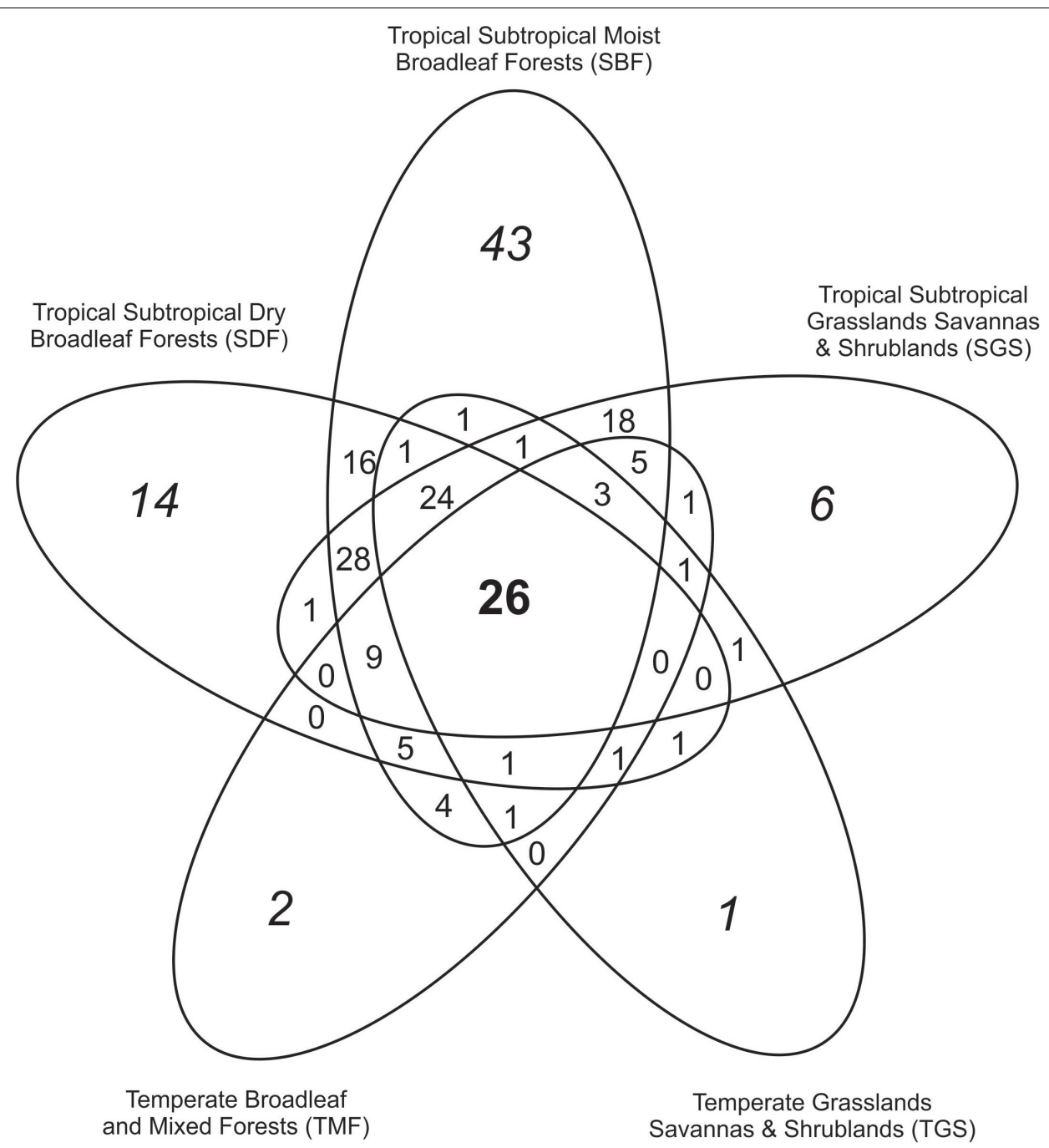

FIGURE 3 | Venn diagram showing the number of species in Glomeromycota that are unique and shared among selected Neotropical biomes.

TABLE 2 | Jaccard's similarity of arbuscular mycorrhizal fungi species between biomes of the Neotropical biogeography realm.

\begin{tabular}{|c|c|c|c|c|c|c|c|c|c|c|}
\hline & DXS & MAN & MED & MON & TMF & TGS & SDF & SGS & SBF & FGS \\
\hline MED & 0.23 & 0.22 & & & & & & & & \\
\hline TMF & 0.16 & 0.27 & 0.50 & 0.23 & & & & & & \\
\hline TGS & 0.15 & 0.30 & 0.24 & 0.22 & 0.37 & & & & & \\
\hline SDF & 0.13 & 0.33 & 0.22 & 0.18 & 0.28 & 0.40 & & & & \\
\hline FGS & 0.12 & 0.20 & 0.13 & 0.21 & 0.16 & 0.21 & 0.15 & 0.15 & 0.10 & \\
\hline SCF & 0.15 & 0.30 & 0.15 & 0.15 & 0.23 & 0.33 & 0.27 & 0.23 & 0.20 & 0.20 \\
\hline
\end{tabular}

See Figure 2 for abbreviation of biomes.

Most of AMF species in the Neotropics were recorded in five biomes (TMF, TGS, SDF, SGS, and SBF) which had the largest number of records in the $\mathrm{BD}$, showing clearly a bias of studies toward these biomes. Mangroves, Flooded Grasslands, and Desert and Xeric Shrublands are biomes underrepresented in the $\mathrm{BD}$, and further studies in these 


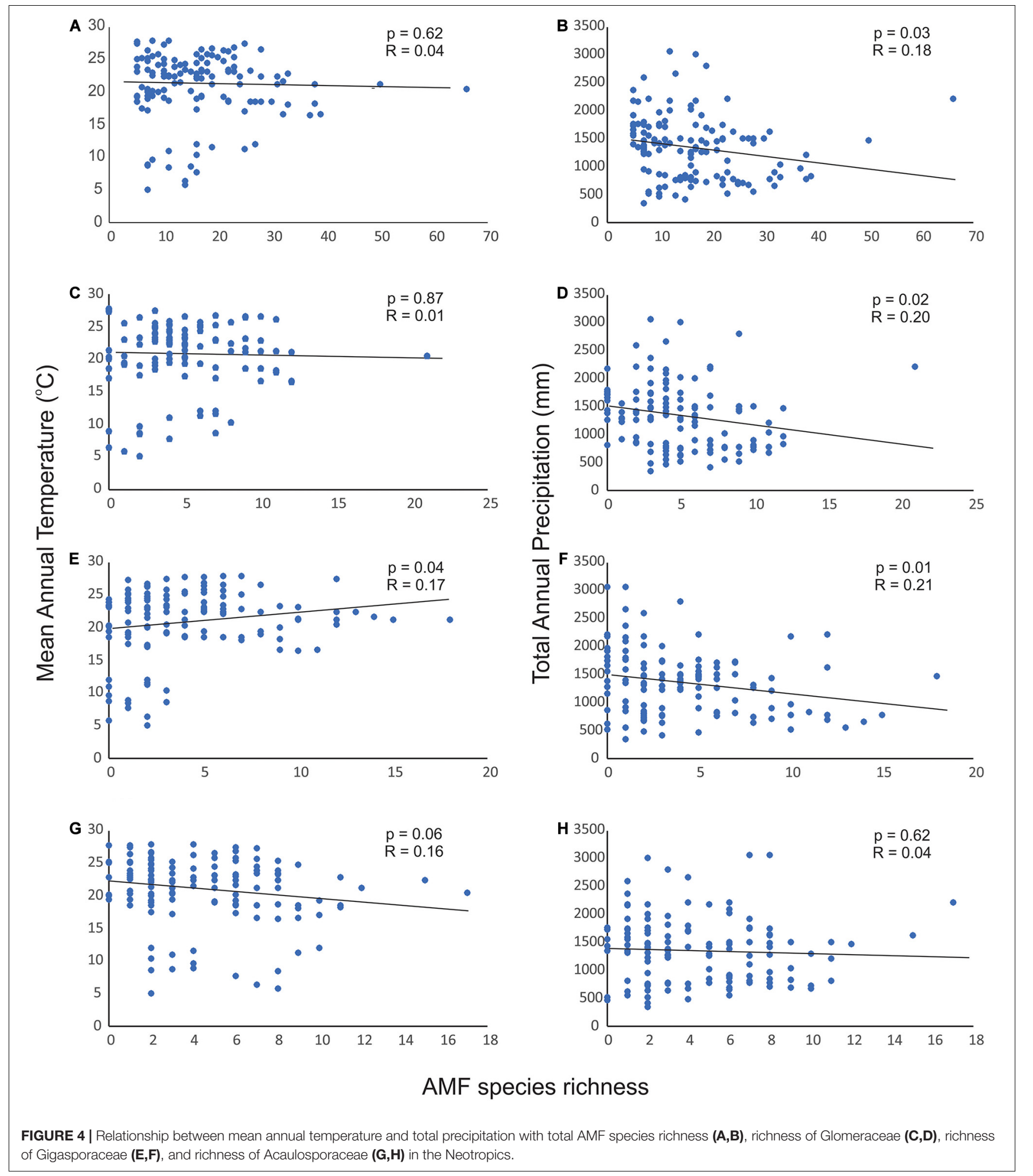

biomes will certainly contribute to our knowledge on AMF distribution in the Neotropics. Only a small set (26 species) of the total number of species in the Neotropics were shared among these five biomes, and they pertained to families that are basal and derived within the Glomeromycota phylogeny. This possibly indicates a characteristic of AMF assemblages: they are composed by species pertaining to basal and derived clades of Glomeromycota, regardless of the geographical location 
and environmental condition. However, this hypothesis has to be further tested more rigorously. Higher similarity among all Neotropical biomes was detected among SBF, SGS, and SDF, all of them being located above the $35^{\circ}$ of latitude South, bordering each other, and with the larger number of studies and species recorded. On average, similarity among biomes within the Neotropics was $25 \%$ which is lower than the average value of similarity among all terrestrial biomes (43 to 56\%) calculated by Davison et al. (2015); Stürmer et al. (2018). A possible reason for this lower similarity among biomes within the Neotropics lies on the Jaccard's index which considers the total number of species detected in one biome. Considering that some biomes were less surveyed for AMF (e.g., MON, MED), their lower number of species decreases the similarity when compared with other biomes with higher number of species.

The relationship between AMF diversity and richness to precipitation and temperature is important considering the global change scenarios (Van der Heijden et al., 2008) and the influence of AMF diversity on plant community diversity and productivity (Van der Heijden et al., 1998). Under field conditions, precipitation had a weak direct effect upon AMF richness but it influenced plant cover which in turn affected AMF species richness (Zhang et al., 2016). Experimental trials showed the increased precipitation, but not warming, decreased AMF OTU richness by $16 \%$ on average compared to control treatments (Gao et al., 2016). Manipulation of rainfall regime for 2 years affected the composition of AMF community but not species richness (Deveautour et al., 2018), although responses of AMF community might take longer time to be observed (Deveautour et al., 2020). These studies were developed mostly in arid and semi-arid grasslands at the regional level, and our results at the scale of a biogeographic realm corroborates that both precipitation and mean annual temperature have a weak influence on AMF species richness in natural communities in the Neotropics. Both climatic data also did not influence species richness of the main families within Glomeromycota, although a significant but weak relationship was found between climatic data and species richness in Glomeraceae and Gigasporaceae. One reason for this lack of relationship might be the narrow range of precipitation and temperature occurring in the Neotropics (e.g., $68 \%$ of temperature values range between 20 and $28^{\circ} \mathrm{C}$ ). Our results also suggest that other variables are more important in the Neotropics to determine AMF richness, such as abiotic factors like soil $\mathrm{P}$ and $\mathrm{pH}$, and biotic factors like the plant community. Considering that AMF communities are highly unpredictable (Powell and Bennett, 2016), species richness might be more influenced by random process or fungal dispersal capacity rather than climatic processes.

In conclusion, the Glomeromycota is well represented in Neotropical biomes and ecoregions and this biogeographic realm is a source for new species and supraspecific taxa which certainly contributes to our knowledge of the fungal dimension of biodiversity. Considering that anthropogenic actions are a threat to biodiversity in the Neotropics, assessment of AMF species composition in understudied ecoregions and biomes is urgent not only to detect new species and occurrences but also to establish these fungi in single-species culture for further use. In this point, we agree with Cofré et al. (2019) that it is important to generate species inventories from Neotropics ecosystems and obtain and deposit single culture in culture collections. Some patterns detected herein among biomes might be biased due to the few studies that have been conducted in some biomes and ecoregions. Indeed, most studies and species have been reported from Tropical and Subtropical Forests, Tropical and Subtropical Grasslands, and Tropical Dry Forests. Some biodiversity hotspots recognized by Myers et al. (2000) like Central Chile, Tropical Andes, and Western Ecuador are scarcely surveyed for AMF communities and efforts should be made to inventory Glomeromycota in these regions. Not only might results from these understudied hotspots reveal new species to science but also it provides information to subsidies public policies for conservation. Assessment of Glomeromycota biodiversity in distinct ecoregions of the Neotropics should be encouraged considering that these fungi play important roles that help to maintain terrestrial ecosystems.

\section{DATA AVAILABILITY STATEMENT}

The raw data supporting the conclusions of this article will be made available by the authors, without undue reservation.

\section{AUTHOR CONTRIBUTIONS}

SS managed the database, compiled Excel spreadsheets, and wrote the manuscript. KK analyzed the data, compiled species list and authority, and edited the manuscript. Both authors approved the final version of the manuscript.

\section{ACKNOWLEDGMENTS}

We thank André L. de Gasper for helping with climatic data and review of early version of the manuscript. Caroline Krug Vieira and Chaiane Schoen for assistance with statistical analyses. We are in debt with Coline Deveautour for some discussion and ideas on AMF communities. We thank two reviewers for their comments to improve the manuscript. SS would like to thank the CNPq for a Research Assistantship (Process 307.995/2019-4).

\section{SUPPLEMENTARY MATERIAL}

The Supplementary Material for this article can be found online at: https://www.frontiersin.org/articles/10.3389/fmicb. 2020.553679/full\#supplementary-material 


\section{REFERENCES}

Aguilera, P., Cornejo, P., Borie, F., Barea, J. M., von Baer, E., and Oehl, F. (2014). Diversity of arbuscular mycorrhizal fungi associated with Triticum aestivum L. plants growing in an Andosol with high aluminum level. Agric. Ecosyst. Environ. 186, 178-184. doi: 10.1016/j.agee.2014. 01.029

Antonelli, A., and Sanmartín, I. (2011). Why are there so many plant species in the Neotropics? Taxon 60, 403-414. doi: 10.1002/tax.602010

Bentivenga, S. P., and Morton, J. B. (1995). A monograph of the genus Gigaspora, incorporating developmental petterns of morphological characters. Mycologia 87, 719-731. doi: $10.2307 / 3760818$

Bills, R. J., and Morton, J. B. (2015). A combination of morphology dn $28 \mathrm{~S}$ rRNA gene sequences provide grouping and ranking criteria to merge eight intro three Ambispora species (Ambisporaceae, Glomeromycota). Mycorrhiza 25, 485-498. doi: 10.1007/s00572-015-0626-7

Blaszkowski, J., Chwat, G., Kovács, G. M., Gáspár, B. K., Ryszka, P., Orlowska, E., et al. (2013). Septoglomus fuscum and S. furcatum, two new species of arbuscular mycorrhizal fungi (Glomeromycota). Mycologia 105, 670-680. doi: $10.3852 / 12-127$

Brown, J. H., and Lomolino, M. V. (2008). Biogeography. Sunderland: Sinauer Associates Inc.

Brundrett, M. C., and Tedersoo, L. (2018). Evolutionary history of mycorrhizal symbioses and global host plant diversity. New Phytol. 220, 1108-1115. doi: 10.1111/nph.14976

Carrenho, R., Silva, E. S., Trufem, S. F. B., and Bononi, V. L. R. (2001). Successive cultivation of maize and agricultural practices on root colonization, number of spores and species of arbuscular mycorrhizal Fungi. Braz. J. Microbiol. 32, $262-270$.

Carvalho, F., De Souza, F. A., Carrenho, R., Moreira, F. M. S., Jesus, E. C., and Fernandes, G. W. (2012). The mosaic of habitats in the high-altitude Brazilian rupestrian fields is a hotspot for arbuscular mycorrhizal Fungi. Appl. Soil. Ecol. 52, 9-19. doi: 10.1016/j.apsoil.2011.10.001

Castillo, C., Borie, F., Godoy, R., Rubio, R., and Sieverding, E. (2005). Diversity of mycorrhizal plant species and arbuscular mycorrhizal fungi in evergreen forest, deciduous forest and grassland ecosystems of Southern Chile. J. Appl. Bot. Food Qual. 80, 40-47.

Chazot, N., Willmott, K. R., Lamas, G., Freitas, A. V. L., Piron-Prunier, F., Arias, C. F., et al. (2019). Renewed diversification following miocene landscape turnover in a neotropical butterfly radiation. Global Ecol. Biogeogr. 28, 1118 1132. doi: $10.1111 /$ geb.12919

Cofré, M. N., Soteras, F., Iglesias, M. R., Velázquez, S., Abarca, C., Risio, L., et al. (2019). "Biodiversity of arbuscular mycorrhizal fungi in south america: a review," in Mycorrhizal Fungi in South America, eds M. C. Pagano and M. A. Lugo (Berlin: Springer), 49-72. doi: 10.1007/978-3-030-15228-4_3

Coutinho, E. S., Fernandes, G. W., Berbara, R. L. L., Valeìrio, H. M., and Goto, B. T. (2015). Variation of arbuscular mycorrhizal fungal communities along an altitudinal gradient in rupestrian grasslands in Brazil. Mycorrhiza 25, 627-638. doi: 10.1007/s00572-015-0636-5

Cuenca, G., and Lovera, M. (1992). Vesicular-arbuscular mycorrhizae in disturbed and revegetated sites from La Gran Sabana. Venezuela. Can. J. Bot. 70, 73-79. doi: 10.1139/b92-009

Davison, J., Moora, M., Öpik, M., Adholeya, A., Ainsaar, L., Bâ, A., et al. (2015). Global assessment of arbuscular mycorrhizal fun- gus diversity reveals very low endemism. Science 349, 970-973. doi: 10.1126/science.aab1161

Deveautour, C., Donn, S., Power, S. A., Bennett, A. E., and Powell, J. R. (2018). Experimentally altered rainfall regimes and host root traits affect grasslands arbuscular mycorrhizal fungal communities. Mol. Eco. 27, 2152-2163. doi: 10.1111/mec. 14536

Deveautour, C., Power, S. A., Barnett, K. L., Ochoa-Hueso, R., Donn, S., Bennett, A. E., et al. (2020). Temporal dynamics of mycorrhizal fungal communities and co-associations with grassland plant communities following experimental manipulation of rainfall. J. Ecol. Soc. 108, 515-527. doi: 10.1111/1365-2745. 13267

Dotzler, N., Krings, M., Taylor, T. N., and Agerer, R. (2006). Germination shields in Scutellospora (Glomeromycota: Diversisporales, Gigasporaceae) from the 400 million-year-old Rhynie chert. Mycol. Prog. 5, 178-184. doi: 10.1007/s11557006-0511-z
Dotzler, N., Walker, C., Krings, M., Hass, H., Kerp, H., Taylor, T. N., et al. (2009). Acaulosporoid glomeromycotan spores with a germination shield from the 400million-year-old Rhynie chert. Mycol. Prog. 8, 9-18. doi: 10.1007/s11557-0080573-1

Escudero, V., and Mendoza, R. (2005). Seasonal variation of arbuscular mycorrhizal fungi in temperature grassland along a wide hydrologic gradient. Mycorrhiza 15, 291-299. doi: 10.1007/s00572-004-0332-3

Gao, C., Kim, Y.-C., Zheng, Y., Yang, W., Chen, L., Ji, N.-N., et al. (2016). Increased precipitation, rather than warming, exerts a strong influence on arbuscular mycorrhizal fungal community in a semiarid steppe ecosystem. Botany 94, 459-469. doi: 10.1139/cjb-2015-0210

Gentry, A. H. (1982). Neotropical floristic diversity: phytogeographical connections between Central and South America, Pleistocene climatic fluctuations, or an accident of the andean orogeny? Ann. Miss. Bot. Garden. 69, 557-593. doi: 10.2307/2399084

Goto, B. T., da Silva, G. A., Yano-Melo, A. M., and Maia, L. C. (2010). Checklist of the arbuscular mycorrhizal fungi (Glomeromycota) in the Brazilian semiarid. Mycotaxon 113, 251-254. doi: 10.5248/113.251

Goto, B. T., Silva, G. A., Assis, D. M. A., Silva, D. K. A., Souza, R. G., Ferreira, A. C. A., et al. (2012). Intraornatosporaceae (Gigasporales), a new family with two new genera and two new species. Mycotaxon 119, 117-132. doi: 10.5248/ 119.117

Hammer, Ø, Harper, D. A. T., and Ryan, P. D. (2001). PAST: paleontological statistics software package for education and data analysis. Palaeontol. Electron. 4, 1-9.

Hart, M. M., and Reader, R. J. (2002). Taxonomic basis for variation in the colonization strategy of arbuscular mycorrhizal fungi. New Phytol. 153, 335344. doi: 10.1046/j.0028-646x.2001.00312.x

Holt, B. G., Lessard, J. P., Borregaard, M. K., Fritz, S. A., Araújo, M. B., Dimitrov, D., et al. (2013). An update of Wallace's zoogeographical regions of the world. Science 339, 74-78.

Hoorn, C., Wesselingh, F. P., ter Steege, H., Bermudez, M. A., Mora, A., Sevink, J., et al. (2010). Amazonia through time: andean uplift, climate change, landscape evolution, and biodiversity. Science 330, 927-931. doi: 10.1126/science. 1194585

International Culture Collection of Glomeromycota [CICG] (2020). International Culture Collection of Glomeromycota. Available online at: https://sites.google. com/site/cicgfma/home (Acessed November 17, 2020).

Jobim, K., Blaszkowski, J., Niezgoda, P., Kozlowska, A., Zubek, S., Mleczko, S., et al. (2019). New sporocarpic taxa in the phylum Glomeromycota: Sclerocarpum amazonicum gen. et sp. nov. in the family Glomeraceae (Glomerales), and Diversispora sporocarpia sp. nov. in the Diversisporaceae (Diversisporales). Mycol. Prog. 18, 369-384. doi: 10.1007/s11557-018-01462-2

Jobim, K., Oliveira, B. I. S., and Goto, B. T. (2016). Checklist of the Glomeromycota in the Brazilian Savanna. Mycotaxon 131, 1-13. doi: 10.1007/978-3-319-235349_1

Jobim, K., Vista, X. M., and Goto, B. T. (2018). Updates on the knowledge of arbuscular mycorrhizal fungi (Glomeromycotina) in the Atlantic Forest biome - an example of very high species richness in Brazilian biomes. Mycotaxon 133, 1-17

Lugo, M. A., and Cabello, M. N. (2002). Native arbuscular mycorrhizal fungi (AMF) from mountain grassland (Coìrdoba, Argentina) I. Seasonal variation of fungal spore diversity. Mycologia 94, 579-586. doi: 10.2307/3761709

Lugo, M. A., Ferrero, M. A., Menoyo, E., Esteivez, M. C., Sinperiz, F., and Anton, A. M. (2008). Arbuscular mycorrhizal fungi and rhizospheric bacteria diversity along an altitudinal gradient in South American Puna grassland. Microb. Ecol. 55, 705-713. doi: 10.1007/s00248-007-9313-3

Maia, L. C., Passos, J. H., Silva, J. A., Oehl, F., and Assis, D. M. A. (2020). Species diversity of glomeromycota in brazilian biomes. Sydowia 72, 181-205.

Moreira, M., Baretta, D., Tsai, S. M., Gomes-da-Costa, S. M., and Cardoso, E. J. B. N. (2007). Biodiversity and distribution of arbuscular mycorrhizal fungi in Araucaria angustifolia forest. Sci. Agric. 64, 393-399. doi: 10.1590/s010390162007000400010

Myers, N., Mittermeier, R. A., Mittermeier, C. G., Fonseca, G. A. B., and Kent, J. (2000). Biodiversity hotspots for conservation priorities. Nature 403, 853-858. doi: $10.1038 / 35002501$

Olson, D. M., Dinerstein, E., Wikramanayake, E. D., Burges, N. D., Powell, G. V. N., Underwood, E. C., et al. (2001). Terrestrial ecoregions of the world: a new 
map of life on Earth. BioScience 51, 933-938. doi: 10.1641/0006-3568(2001) 051[0933:teotwa]2.0.co;2

Parenti, L. R., and Ebach, M. C. (2009). Comparative Biogeography-Discovering and Classifying Biogeographical Patterns of a Dynamic Earth. Berkeley: Univ of California Press.

Pereira, C. M. R., da Silva, D. K. A., Ferreira, A. C. A., Goto, B. T., and Maia, L. C. (2014). Diversity of arbuscular mycorrhizal fungi in Atlantic forest areas under different land uses. Agric. Ecosyst. Environ. 185, 245-252. doi: 10.1016/j.agee. 2014.01.005

Pontes, J. S., Oehl, F., Pereira, C. D., Machado, C. T. T., Coyne, D., Silva, D. K. A., et al. (2017). Diversity of arbuscular mycorrhizal fungi in the Brazilian's Cerrado and in soybean under conservation and conventional tillage. Appl. Soil Ecol. 117-118, 178-189. doi: 10.1016/j.apsoil.2017.04.023

Powell, J. R., and Bennett, A. E. (2016). Unpredictable assembly of arbuscular mycorrhizal fungal communities. Pedobiologia 59, 11-15. doi: 10.1016/j. pedobi.2015.12.001

Prance, G. T. (1973). Phytogeographical support for the theory of Pleistocene forest refuges in the Amazon basin, based on evidence from distribution patterns in Caryocaraceae, Chrysobalanaceae, Dichapetalaceae and Lecythidaceae. Acta Amazon. 3, 5-28. doi: 10.1590/1809-43921973033005

Redecker, D., Schussler, A., Stockinger, H., Sturmer, S. L., Morton, J. B., and Walker, C. (2013). An evidence-based consensus for the classification of arbuscular mycorrhizal fungi (Glomeromycota). Mycorrhiza 23, 515-531. doi: 10.1007/ s00572-013-0486-y

Remy, W., Taylor, T. N., Hass, H., and Kerp, H. (1994). Four hundred-millionyear- old vesicular arbuscularmycorrhizae. Proc. Natl. Acad. Sci. U.S.A. 91, 11841-11843. doi: 10.1073/pnas.91.25.11841

Schneider, J., Stürmer, S. L., Guilherme, L. R. G., Moreira, F. M. S., and Soares, C. R. F. S. (2013). Arbuscular mycorrhizal fungi in arsenic-contaminated areas in Brazil. J. Hazard. Mater. 262, 1105-1115. doi: 10.1016/j.jhazmat.2012.09.063

Schussler, A., Mollenhauer, D., Schnepf, E., and Kluge, M. (1994). Geosiphon pyriforme, an endosymbiotic association of fungus and cyanobacteria: the spore structure resembles that of arbuscular mycorrhizal (AM) fungi. Plant Biol. 107, 36-45. doi: 10.1111/j.1438-8677.1994.tb00406.x

Silva, I. R., Mello, C. M. A., Neto, R. A. F., Silva, D. K. A., Melo, A. L., Oehl, F., et al. (2014). Diversity of arbuscular mycorrhizal fungi along an environmental gradient in the Brazilian semiarid. Appl. Soil Ecol. 84, 166-175. doi: 10.1016/j. apsoil.2014.07.008

Siqueira, J. O., Colozzi-Filho, A., and Oliveira, E. (1989). Ocorrência de micorrizas vesicular-arbusculares em agro e ecossistemas do estado de Minas Gerais. Pesq. Agropec. Bras. 24, 1499-1506.

Souza, R. G., da Silva, D. K. A., Mello, C. M. A., Goto, B. T., Silva, F. S. B., Sampaio, E. V. S. B., et al. (2013). Arbuscular mycorrhizal fungi in revegetated mined dunes. Land Degrad. Dev. 24, 147-155. doi: 10.1002/ldr.1113

Stürmer, S. L., and Bellei, M. M. (1994). Composition and seasonal variation of spore populations of arbuscular mycorrhizal fungi in dune soils on the island of Santa Catarina, Brazil. Can. J. Bot. 72, 359-363. doi: 10.1139/b94-048
Stürmer, S. L., Bever, J. D., and Morton, J. B. (2018). Biogeography of arbuscular mycorrhizal fungi (Glomeromycota): a phylogenetic perspective on species distribution patterns. Mycorrhiza 28, 587-603. doi: 10.1007/s00572-0180864-6

Stürmer, S. L., and Siqueira, J. O. (2011). Species richness and spore abundance of arbuscular mycorrhizal fungi across distinct land uses in Western Brazilian Amazon. Mycorrhiza 21, 255-267. doi: 10.1007/s00572-0100330-6

Van der Heijden, M., Klironomos, J., Ursic, M., Moutoglis, P., Streitwolf-Engel, R., Boller, T., et al. (1998). Mycorrhizal fungal diversity determines plant biodiversity, ecosystem variability and productivity. Nature 396, 69-72. doi: $10.1038 / 23932$

Van der Heijden, M. G. A., Bardgett, R. D., and van Straalen, N. M. (2008). The unseen majority: soil microbes as drivers of plant diversity and productivity in terrestrial ecosystems. Ecol. Lett. 11, 296-310. doi: 10.1111/j.1461-0248.2007. 01139. $\mathrm{x}$

Velázquez, M. S., Fabisik, J. C., Abarca, C. L., Allegrucci, N., and Cabello, M. (2016a). Colonization dynamics of arbuscular mycorrhizal fungi (AMF) in Ilex paraguariensis crops: seasonality and influence of management practices. J. King Saud. Univ. 32, 183-188. doi: 10.1016/j.jksus.2018.03.017

Velázquez, M. S., Stürmer, S. L., Bruzone, C., Fontenla, S., Barrera, M., and Cabello, M. (2016b). Occurrence of arbuscular mycorrhizal fungi in high altitude sites of the Patagonian Altoandina region in Nahuel Huapi National Park (Argentina). Acta Bot. Bras. 30, 521-531. doi: 10.1590/0102-33062016abb 0223

Walker, C., Trappe, J. M., Schussler, A., Hawksworth, D. L., Cazares, E., Elliott, T. F., et al. (2017). Proposal to conserve the name Rhizophagus with a conserved type (Fungi:Glomeromycota:Glomeraceae). Taxon 66, 199-200. doi: 10.12705/ 661.19

Wijayawardene, N. N., Hyde, K. D., Dai, D. Q., Tang, L. Z., Aptroot, A., CastañedaRuiz, R. F., et al. (2020). Outline of Fungi and fungi-like taxa. Mycosphere 11, 1060-1456. doi: 10.5943/mycosphere/11/1/8

Zhang, J., Wang, F., Che, R., Wang, P., Liu, H., Ji, B., et al. (2016). Precipitation shapes communities of arbuscular mycorrhizal fungi in Tibetan alpine steppe. Sci. Rep. 6:23488.

Conflict of Interest: The authors declare that the research was conducted in the absence of any commercial or financial relationships that could be construed as a potential conflict of interest.

Copyright (๑) 2021 Stürmer and Kemmelmeier. This is an open-access article distributed under the terms of the Creative Commons Attribution License (CC BY). The use, distribution or reproduction in other forums is permitted, provided the original author(s) and the copyright owner(s) are credited and that the original publication in this journal is cited, in accordance with accepted academic practice. No use, distribution or reproduction is permitted which does not comply with these terms. 